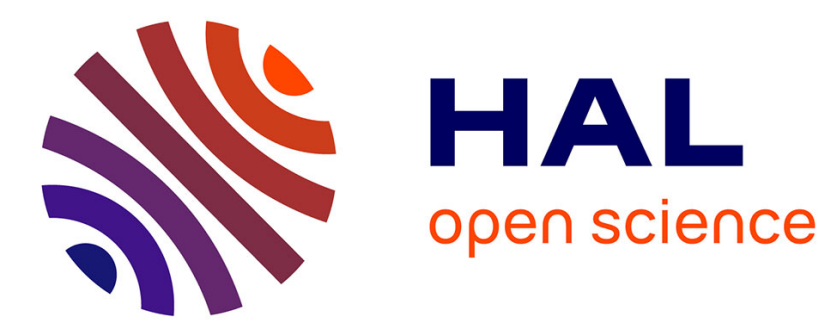

\title{
Organization and structure of mixed Langmuir films composed of polydiacetylene and hemicyanine
}

Gonzalo García-Espejo, Marta Pérez-Morales, Michel Goldmann, María T.

Martín-Romero, Juan J. Giner-Casares, Luis Camacho

\section{- To cite this version:}

Gonzalo García-Espejo, Marta Pérez-Morales, Michel Goldmann, María T. Martín-Romero, Juan J. Giner-Casares, et al.. Organization and structure of mixed Langmuir films composed of polydiacetylene and hemicyanine. Journal of Colloid and Interface Science, 2017, 508, pp.583-590. 10.1016/j.jcis.2017.08.069 . hal-01586785

\section{HAL Id: hal-01586785 \\ https://hal.sorbonne-universite.fr/hal-01586785}

Submitted on 13 Sep 2017

HAL is a multi-disciplinary open access archive for the deposit and dissemination of scientific research documents, whether they are published or not. The documents may come from teaching and research institutions in France or abroad, or from public or private research centers.
L'archive ouverte pluridisciplinaire HAL, est destinée au dépôt et à la diffusion de documents scientifiques de niveau recherche, publiés ou non, émanant des établissements d'enseignement et de recherche français ou étrangers, des laboratoires publics ou privés. 


\author{
Gonzalo García-Espejo ${ }^{1}$, Marta Pérez-Morales ${ }^{1}$, Michel Goldmann ${ }^{2,3}$, María T. Martín-Romero ${ }^{1}$, \\ Juan J. Giner-Casares ${ }^{1 *}$, and Luis Camacho ${ }^{1 *}$ \\ ${ }^{1}$ Institute of Fine Chemistry and Nanochemistry, Department of Physical Chemistry and Applied \\ Thermodynamics, University of Córdoba, Campus Universitario de Rabanales, Edificio Marie \\ Curie, Córdoba, Spain E-14014 \\ ${ }^{2}$ Sorbonne Universite's, UPMC Univ Paris 06, CNRS-UMR 7588, Institut des \\ NanoSciences de Paris, F-75005, Paris, France \\ ${ }^{3}$ Synchrotron SOLEIL L'Orme des Merisiers Saint-Aubin, BP48 91192, \\ Gif/Yvette Cedex, France
}

\begin{abstract}
Mixed Langmuir monolayers of 10,12-Pentacosadiynoic acid (DA) monomer and an amphiphilic Hemicyanine dye derivative have been formed at the air/water interface. Two derivatives of docosylpyridinium have been used, with either one included in the monolayer in 1:1 molar ratio. The DA monomers within the mixed monolayers have been polymerized in situ at the air/water interface. The crystalline structure of the monolayer and the kinetics of polymerization have been probed grazing incidence X-ray diffraction (GIXD). The polymerization of DA proceeds with no phase segregation, exclusively leading to the red polydiacetylene form. The kinetics of polymerization at the air/water interface has been monitored in situ by GIXD. The experimental results have been combined with Molecular Mechanics computer simulations, revealing that DA molecules are sequentially arranged with molecules of Hemicyanine dye in alternating rows. The hydrophobic chains of the dye molecules act as spacers between the DA monomers. Surprisingly, such molecular arrangement does not hinder the in situ photopolymerization of DA. The mechanism of polymerization of DA within the mixed Langmuir monolayers has been convincingly described in molecular detail. This approach for interfacial polymerization of DA holds great potential for optically active devices and nanostructures comprising self-assembled thin films based in polydiacetylene.
\end{abstract}

E-mail: jjginer@uco.es, lcamacho@uco.es

KEYWORDS: Langmuir monolayers; GIXD; diacetylene; air/liquid interface 


\section{Introduction.}

Diacetylene (DA) displays highly promising features thanks to the sensing properties of polydiacetylene (PDA), showing a remarkable optical response. ${ }^{1,2}$ The amphiphilic derivatives of DA are especially interesting as they can be self-assembled onto 3D nanostructures with enhanced optical properties. ${ }^{3-5}$ The stability of the self-assembled PDA structures is superior to similar architectures, $i$. e., liposomes, promoting the application in biomedicine and therapy. ${ }^{6,7}$ Assembly and polymerization of DA onto planar surfaces have rendered interesting materials, with 10,12-pentacosadiynoic acid as a forefront monomer. ${ }^{8,9}$ DA can be polymerized in situ at interfaces using the simple procedure of irradiation with UV light. ${ }^{10,11}$ The Langmuir technique is an advantageous platform for the study in fine detail of the assembly and polymerization processes of DA at air/liquid interfaces. ${ }^{12}$

The backbone of PDA contains alternating double and triple bonds. The backbone can be aligned in a periodic manner with an absorption band centered at ca. $650 \mathrm{~nm}$, being the "blue" PDA phase. The backbone can alternatively display a certain local torsion with no net torsion on the PDA polymer chain, then having the absorption band centered at ca. $550 \mathrm{~nm}$ and being the "red" PDA phase. ${ }^{13}$ The topological features of the PDA are therefore fundamental in the optical properties and the subsequent applications of PDA. Introducing functional groups to PDA nanostructures allows new applications: a mixed PDA surface containing biotin and ethanolamide bound the target, i.e., streptavidin, more specifically than did biotin alone. The optimized PDA biosensor exhibited approximately 2850-fold higher selectivity for streptavidin relative to bovine serum albumin controls. ${ }^{14}$ In this context we have reported that 10,12 pentacosadiynoic acid can be successfully incorporated in mixed Langmuir monolayers with an amphiphilic hemicyanine dye, either 4-[(4-hydroxy)styryl]-1-docosylpyridinium bromide (HSP) or 4-[4-(dimethylamino)styryl]-1-docosyl- pyridinium bromide (SP), see Scheme 1.A. ${ }^{15,16}$ Three main factors contribute to an optimum molecular packing: a) the spatial match of the hydrophobic chains, b) the successful formation of an ion pair between the polar headgroups, c) the correct balance between the effective molecular area of the hydrophobic chains and the polar headgroups of both molecules.

The polymerization of DA is a 1-4 addition with topochemical control in both initiation and growth steps. ${ }^{17}$ Lateral and top views of a DA network adapted from the cell parameters from Lifshitz et al. is shown in scheme $1 \mathrm{~B}, \mathrm{C}{ }^{18}$ The polymerization of DA in bulk solution occurs exclusively along of the direction of $\boldsymbol{r}$ axes, leading to the blue PDA as primary reaction product, see Scheme 1D. ${ }^{19}$ In that case, polymerization along the $\boldsymbol{k}$ axis does not occur. In principle, the polymerization of DA on surfaces, such as mixed Langmuir monolayers should meet the mentioned topochemical condition. Surprisingly, herein we could quantitatively assess that while such requirement is not met by the mixed Langmuir monolayers containing DA due to the molecular organization within the monolayer, the polymerization of DA indeed takes 
place. Using synchrotron grazing incidence X-ray diffraction (GIXD), DA:HSP and DA:SP mixed Langmuir monolayers are studied in detail. In contrast to the bulk polymerization of DA, the mixed Langmuir monolayers containing DA display a polymerization process through different geometric criteria. A similar mechanism to the observed for the 2D photopolymerization of DA adsorbed on graphite is proposed, where the diacetylene units are ordered in a similar way as are placed along the $\boldsymbol{k}$ axis in Scheme $1 .^{20,21}$

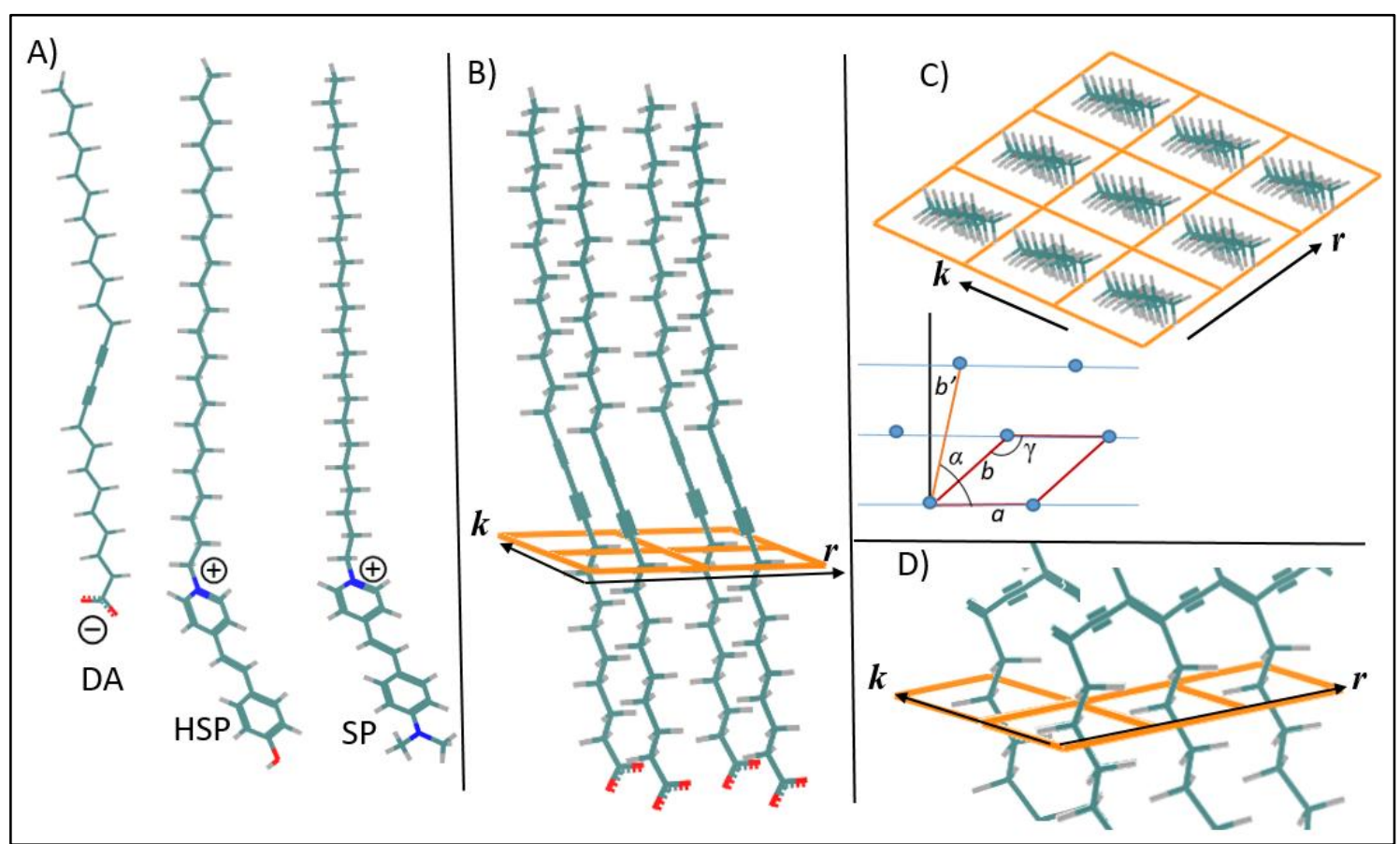

SCHEME 1. (A) Molecular structures of 10,12-pentacosadiynoic acid (DA), 4-[(4hydroxy)styryl]-1-docosylpyridinium bromide (HSP), 4-[4-(dimethylamino)styryl]-1-docosylpyridinium bromide (SP). (B) Lateral and (C) top views of a sketch of pure DA network built from the cell parameters from Lifshitz et al., see Table 1 for values of cell parameters. ${ }^{18}$ Inset in C: geometric criteria for $2 \mathrm{D}$ cell parameters. (D) Topochemical 3D polymerization of DA along the r-axis.

\section{Experimental section}

2.1. Materials.

10,12-Pentacosadiynoic acid (DA) was purchased from ABCR (Germany) and purified as follows: The diacetylene monomer was dissolved in chloroform and filtered through a $0.45 \mu \mathrm{m}$ nylon filter. Purified powder was obtained by evaporation of the solvent. ${ }^{22}$ Hemicyanine dye, 4-[(4-hydroxy)styryl]-1-docosylpyridinium bromide (HSP) and Hemicyanine dye, 4-[4(dimethylamino)styryl]-1-docosyl- pyridinium bromide (SP), were purchased from SigmaAldrich and used as received. The spreading solutions for each component were prepared using chloroform as a solvent. A mixture of trichloromethane and methanol, ratio 3:1 (v/v) was used as cospreading solvent. All mixed Langmuir monolayers composed by two components were 
prepared in 1:1 molar ratio. The pure solvents were obtained from Aldrich (Germany) and used by a Millipore reverse osmosis system ( $>18.2 \mathrm{M} \Omega \square \mathrm{cm}$ ), was used as a subphase. The subphase temperature was in all cases $21^{\circ} \mathrm{C}$, with $\mathrm{pH}$ 5.7.

\subsection{Methods.}

Two different models of NIMA troughs (NIMA Technology, Coventry, England) were used in this work, both provided with a Wilhelmy type dynamometric system using a strip of filter paper: a NIMA 611D with one moving barrier for the measurement of the reflection spectra and a NIMA 601, equipped with two symmetrical barriers to record BAM images. The monolayers were compressed at a speed of $0.03 \mathrm{~nm}^{2} \min ^{-1}$ molecule ${ }^{-1}$. For the UV irradiation a UV lamp ( $\lambda$ $=254 \mathrm{~nm}, 10 \mathrm{~W}$ ) was mounted on top of the trough, keeping a distance of ca. $5 \mathrm{~cm}$ from the mixed Langmuir monolayer.

The GIXD measurements were performed on the SIRIUS beamline at the SOLEIL synchrotron. The details and optics of the facility are described elsewhere. ${ }^{23}$ The incident X-ray energy used was $10.5 \mathrm{keV}(\lambda=0.118 \mathrm{~nm})$ and the beam size was $0.1 \times 1 \mathrm{~mm}^{2}(\mathrm{~V} \times \mathrm{H})$ at the sample position. The water surface was illuminated at an incident angle of $1.8 \mathrm{mrad}$ below the critical angle of the air-water interface $(2.04 \mathrm{mrad}$ at $10.5 \mathrm{keV})$, so that the incident wave was totally reflected, while the refracted wave became evanescent, exploring a layer of several nanometers beneath the interface. The scattered intensity was collected with a low noise, position sensitive, 1D gas detector, with 2048 channels on $150 \mathrm{~mm}$. A custom-built Langmuir trough was enclosed in a temperature controlled, sealed chamber and flushed with helium during data collection to reduce gas scattering and to avoid beam damage to the monolayer. A quartz window allows the vertical UV irradiation of the film without opening the sealed through maintaining then the helium atmosphere. GIXD was used to obtain in-plane information about the molecular structure of the surface. The spectra were obtained by varying the X-ray, momentum transfer, in-plane component $\mathrm{q}_{\mathrm{xy}}$ that is parallel to the air-water interface. The scattered intensity was measured as a function of the angle, $2 \theta$, between the incident and diffracted beam projected onto the horizontal plane.

2.3 Computer Simulations.

Semiempirical PM3 methods for geometry optimization of DA, HSP and SP units were used. ${ }^{24}$ The optimized structures are place in $2 \mathrm{D}$ periodic box of predefined size, according the simulated system. Starting from different initial positions, the structures were optimized by using COMPASS method. ${ }^{25}$ 


\section{3) Grazing incidence diffraction experiments.}

\section{Previous reports on polymerization of DA onto PDA at the air/liquid interface.}

Previous GIXD studies on Langmuir monolayers containing DA provide results that are not completely consistent, probably due to different experimental conditions. Note that a slight polymerization of the DA monolayer might take place, given the irradiation with X-ray light during GIXD experiments. Despite the DA monolayer on pure water is not stable, the stability is improved by spreading on a basic subphase. ${ }^{26}$ Therefore, Gourier et al. performed the experiments on a subphase with $\mathrm{pH}=7.5$ using a sodium tetraborate buffer. Moreover, filtered DA solutions were used to remove residual polymer present in the commercial compound. ${ }^{27}$ Such experimental conditions for the non-polymerized monolayer at $20 \mathrm{mN} / \mathrm{m}\left(22^{\circ} \mathrm{C}\right)$ leads to two $q_{\mathrm{xy}}$ diffraction peaks at $q_{\mathrm{xy}}=1.38 \AA^{-1}$, and $q_{\mathrm{xy}}=1.50 \AA^{-1}$, see Table 1 . The diffraction pattern is consistent with an oblique cell of $a=5.13 \AA, b=4.72 \AA$ ( $b^{\prime}=8.88 \AA$ ), $\gamma=117.4^{\circ}$, the cell parameters defined in Figure 1C. The area per unit cell is $A=21.5 \AA^{2}$. The polymerized monolayer composed by PDA displays two diffraction peaks at $q_{\mathrm{xy}}=1.47$ and $q_{\mathrm{xy}}=1.54 \AA^{-1}$, plus one significantly weaker at $q_{\mathrm{xy}}=2.55 \AA^{-1}$. The dimensions of the associated oblique unit cell are $a=5.05 \AA, b=4.81 \AA, \gamma=122^{\circ}$ and $A=20.5 \AA^{2}$. Although no data on the optical features of the PDA were provided, taking into account the $\mathrm{pH}$ of the subphase as commented above the red form of PDA is assumed.

Lifshitz et al. performed the GIXD experiments on water subphase at $25 \mathrm{mN} / \mathrm{m}$, experimental conditions for producing a trilayer of DA. ${ }^{18}$ The structure of the film was described as two sublayers: a) the hydrophobic methyl-terminated alkyl and b) hydrophilic carboxyl-terminated alkyl chains. The two sublayers are attached on different sides of the diacetylene polymer backbone. The $q_{\mathrm{xy}}$ diffraction peaks were classified into two groups: one group with much weaker signal and values of $q_{\mathrm{z}}>0.8 \AA^{-1}$ (hydrophobic sublayer), and another group with comparatively stronger signal and $q_{\mathrm{z}}<0.5 \AA^{-1}$ (hydrophilic sublayer) in contact with the aqueous subphase. The peaks at $q_{\mathrm{z}}<0.5 \AA^{-1}$ are exclusively analyzed here, see Table 1 . One diffraction peak at $q_{\mathrm{xy}}=1.37 \AA^{-1}$ was obtained for the DA monolayer prior to polymerization. This diffraction pattern is consistent with a hexagonal structure which can be also described with a two molecules rectangular cell with $a=5.27 \AA$, $b^{\prime}=9.13 \AA, \alpha=90^{\circ}\left(\gamma=120^{\circ}\right), A=24.05$ $\AA^{2}$, and no tilting of the hydrophilic chains. Concerning the PDA polymer at the air/water interface, the two forms of PDA were considered. For the blue PDA phase, three $q_{\mathrm{xy}}$ diffraction peaks with $q_{\mathrm{z}}<0.5 \mathrm{~A}^{-1}$ are observed: $q_{\mathrm{xy}}=1.3 \AA^{-1}, 1.38 \AA^{-1}$, and, $1.49 \AA^{-1}$. The blue phase resulting cell is centered-oblique with $a=4.9 \AA, b^{\prime}=9.73 \AA(b=5.63 \AA), \alpha=85^{\circ}\left(\gamma=120.7^{\circ}\right)$ and $A=23.73 \mathrm{~A}^{2} /$ molecule. In this case the tilt angles of the carboxyl terminated sublayers are ca. $18^{\circ}$. For the red PDA phase, ${ }^{18}$ three diffraction peaks, with $q_{\mathrm{z}}<0.5 \mathrm{~A}^{-1}$ were found: $q_{\mathrm{xy}}=1.45$ $\AA^{-1}, 1.59 \AA^{-1}$, and, $1.61 \AA^{-1}$. In this case, the resulting cell is a centered quasi-rectangular cell with $a=4.9 \AA, b^{\prime}=7.82 \AA(b=4.83 \AA), \alpha=85^{\circ}\left(\gamma=126.3^{\circ}\right)$ and $A=19.2 \mathrm{~A}^{2} /$ molecule. The 
blue-to-red transition was described as a decrease in the area per unit cell, as well as an upright, near-vertical, positioning of both the alkyl and alkanoic residues.

Table 1. Reported values for in-plane $q_{\mathrm{xy}}$ of GIXD diffraction peaks, cell parameters, $a$, $b$ (in $\AA$ ), $\gamma$ and $A$ (in $\AA^{2}$ )

\begin{tabular}{|c|c|c|c|}
\hline & DA & Blue PDA & Red PDA \\
\hline \multirow[t]{2}{*}{ Gourier et $a l^{27}$} & $q_{\mathrm{xy}}=1.38,1.5$ & & $1.47,1.54$ \\
\hline & $\begin{array}{l}a=5.13, b=4.72 \\
\gamma=117.4^{\circ}, \\
A=21.5 .\end{array}$ & & $\begin{array}{l}a=5.05, b=4.81, \\
\gamma=122^{\circ}, \\
A=20.5\end{array}$ \\
\hline \multirow{2}{*}{$\begin{array}{l}\text { Lifshitz et al. }{ }^{18} \\
q_{\mathrm{z}}<0.5 \AA^{-1}\end{array}$} & $q_{\mathrm{xy}}=1.37$ & $1.3,1.38,1.49$ & $1.45,1.59,1.61$ \\
\hline & $\begin{array}{l}a=b=5.27 \\
\gamma=120^{\circ}, \\
A=24.05\end{array}$ & $\begin{array}{l}a=4.9, b=5.63, \\
\gamma=120.7^{\circ}, \\
A=23.73\end{array}$ & $\begin{array}{l}a=4.9, b=4.83 \\
\gamma=126.3^{\circ}, \\
A=19.2\end{array}$ \\
\hline
\end{tabular}

\section{Pure SP and HSP component.}

The surface pressure-molecular area isotherms of the amphiphilic hemicyanine SP monolayer can be tuned by using different ions in the aqueous subphase. ${ }^{28}$ The $\mathrm{H}$-aggregation of the hemicyanine group is significantly influenced by such ions. Brewster angle microscopy (BAM) reveal an inhomogeneous morphology ${ }^{29}$ and no GIXD diffraction peaks were obtained for the SP Langmuir monolayer at all surface pressure..

Likewise, the BAM images of the amphiphilic hemicyanine dye HSP monolayer reveal inhomogeneous films. ${ }^{16}$ However, three diffraction peaks at $q_{\mathrm{xy}}=1.38 \AA^{-1}, 1.465 \AA^{-1}$, and, 1.577 $\AA^{-1}$ were obtained by GIXD for $\pi=30 \mathrm{mN} / \mathrm{m}$, see Figure 1 and Table 2. Assuming a single structure, a rectangular unit cell with $a=5.32 \AA, b=4.93 \AA, \gamma=121.8^{\circ}$ and $A=19.9 \AA^{2}$ is obtained. ${ }^{30}$ Note the area per HSP molecule (ca. $20 \AA^{2}$ ) is significantly different from that observed in the isotherm (ca. $40 \AA^{2}$ at a surface pressure of $30 \mathrm{mN} / \mathrm{m}$ ).

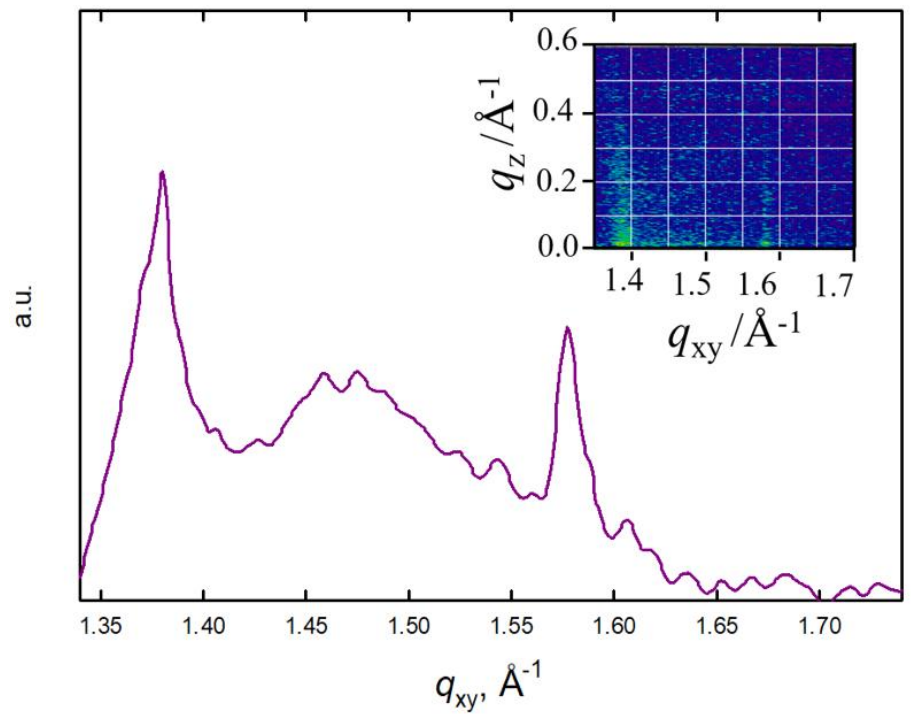

FIGURE 1. GIXD diffraction peaks for the HSP monolayer at $30 \mathrm{mN} / \mathrm{m}$. Inset: Diffraction intensities versus the in-plane and out-of-plane scattering vector component $q_{\mathrm{xy}}$ and $q_{\mathrm{z}}$. 
TABLE 2: In-Plane $q_{\mathrm{xy}}\left(\AA^{-1}\right)^{\mathrm{a}}$ and Out-of-Plane $q_{\mathrm{z}}\left(\right.$ in $\left.\AA^{-1}\right)$ Components of the Scattering Vector and cell parameters of the HSP Monolayer at $T=21^{\circ} \mathrm{C}$ and $\pi=30 \mathrm{mN} / \mathrm{m}$

\begin{tabular}{l|l} 
HSP & $q_{\mathrm{xy} 1}=1.378(0.028), q_{\mathrm{z} 1}=0$
\end{tabular}

$q_{\mathrm{xy} 2}=1.47(0.133), q_{\mathrm{z} 2}=0.43$

$q_{\mathrm{xy} 3}=1.587(0.018), q_{\mathrm{z} 3}=0.43$

$a=5.31 \AA$
$b^{\prime}=7.97$
$\alpha=86.16^{\circ}$

$\mathrm{A}=21.1 \AA^{2}$

$\left.{ }^{a}\right)$ The full-width at half-maximum (FWHM) of the peaks are given in brackets

\section{Mixed Langmuir monolayer DA:HSP.}

Both amphiphilic hemicyanine dye HSP and SP are able to form homogeneous mixed Langmuir monolayers with amphiphilic anionic molecules, such as 10,12-Pentacosadiynoic acid (DA), ${ }^{15,16}$ and phospholipid (DMPA). ${ }^{29}$

The mixed monolayer DA:HSP displays a phase transition at $c a .15 \mathrm{mN} / \mathrm{m} .{ }^{16}$ Prior to the phase transition, no micrometric structures were observed by BAM, whereas domains that grow with further increase of the surface pressure are detected after the phase transition, see inset in Figure 2. No GIXD signal was detected below $15 \mathrm{mN} / \mathrm{m}$, before the phase transition. However, at higher values of surface pressure, after the phase transition and coincidentally with the bright domains, a significant GIXD signal was detected. Note the GIXD information at the nanometric scale is complementary to the BAM domain morphology at the micrometric level.

At $\pi=35 \mathrm{mN} / \mathrm{m}, 2$ diffraction peaks, at $q_{\mathrm{xy}}=1.46,1.56 \AA^{-1}$, and a shoulder at $1.44 \AA^{-1}$ were obtained, see Figure 2 and Table 2. Assuming a single ordered phase, this diffraction pattern is consistent with a triclinic cell of $a=4.81 \AA, b=5.15 \AA\left(b^{\prime}=8.82 \AA\right), \gamma=123.5^{\circ}$, and an area per unit cell, $A=20.7 \AA^{2}$. The X-ray intensities as a function of the scattering vector components $q_{\mathrm{xy}}$ and $q_{\mathrm{z}}$ for the mixed Langmuir monolayer DA:HSP is shown in Figure 3A. The hydrophobic chains are located perpendicular to the interface $\left(q_{\mathrm{z}} \approx 0\right)$.

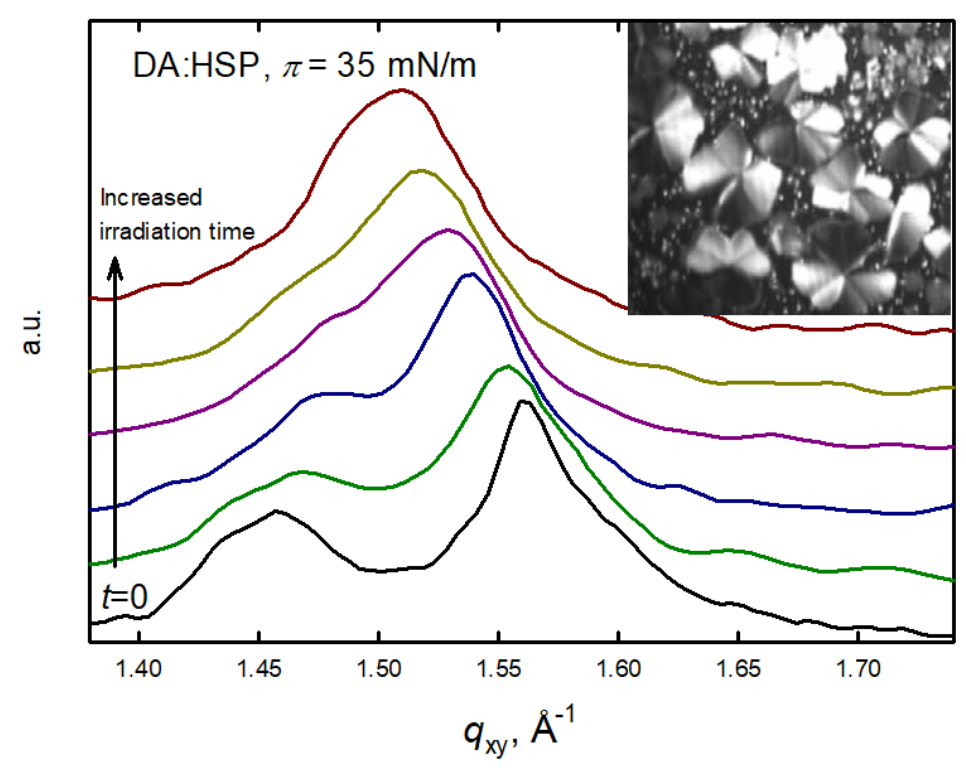


FIGURE 2. Variation of GIXD diffraction peaks for the mixed Langmuir monolayer DA:HSP
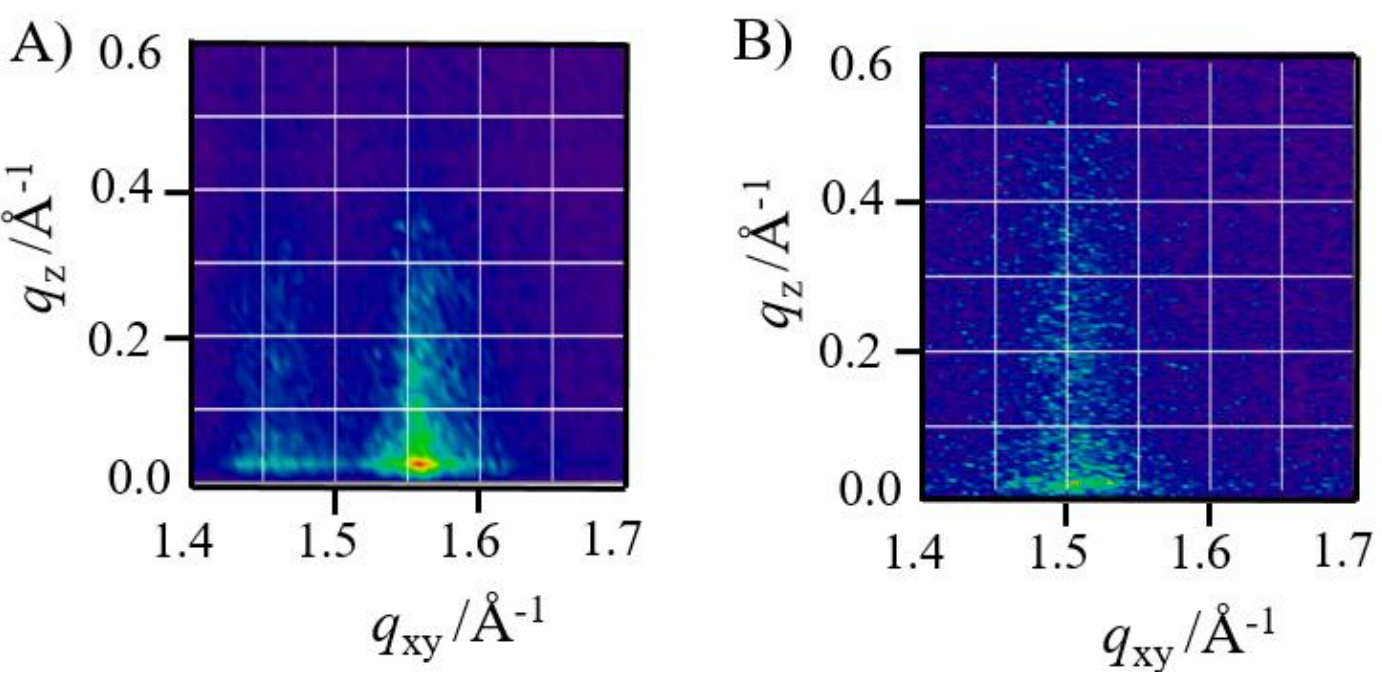

FIGURE 3. X-ray intensities versus the in-plane and out-of-plane scattering vector component $q_{\mathrm{xy}}$ and $q_{\mathrm{z}}$, for the mixed Langmuir monolayer DA:HSP at $35 \mathrm{mN} / \mathrm{m}$. (A) before polymerization, and (B) after the polymerization.

The thickness of the diffracting plane $\left(L_{z}\right)$ can be estimated from the full width at halfmaximum (FWHM) of the Bragg rods by the following equation: $L_{\mathrm{z}} \approx 0.9(2 \pi) / \operatorname{FWHM}\left(q_{\mathrm{z}}\right)$. From the Bragg rods (Table 1), the thickness of the scattering centers, the alkyl chains, would be ca. $26 \AA$ A Assuming an all-trans conformation for the hydrocarbon chains, the length of a alkyl chain $(\mathrm{C} 22)$ from the HSP will be $L_{\max }=(1.26 \times 21+1.5)=28 \AA$. Thus, a good agreement is obtained between the expected thickness of the monolayer and the experimental value obtained by GIXD.

TABLE 3: In-Plane $q_{\mathrm{xy}}\left(\AA^{-1}\right)^{\mathrm{a}}$ and Out-of-Plane $q_{\mathrm{z}}\left(\mathrm{in} \AA^{-1}\right)$ Components of the Scattering 


\begin{tabular}{|c|c|c|c|c|c|}
\hline \multicolumn{6}{|c|}{ Vector and cell parameters for DA:HSP and PDA:HSP system at $T=21^{\circ} \mathrm{C}$} \\
\hline & & $a, \AA$ & $b^{\prime}(b), \AA$ & $\gamma$ & $A, \AA^{2}$ \\
\hline DA/HSP & $\begin{array}{l}q_{\mathrm{xy} 1}=1.436(0.033), q_{\mathrm{z} 1}=0 \\
q_{\mathrm{xy} 2}=1.462(0.044), q_{\mathrm{z} 2}=0 \\
q_{\mathrm{xy} 3}=1.565(0.03), q_{\mathrm{z} 3}=0(0.22)\end{array}$ & 4.76 & $8.74(5.14)$ & $121.9^{\circ}$ & 20.8 \\
\hline PDA/HSP & $\begin{array}{l}q_{\mathrm{xy} 1}=1.488(0.05) \\
q_{\mathrm{xy} 2}=1.516(0.05) \\
q_{\mathrm{z} 1}=0(0.2)\end{array}$ & 4.91 & $8.30(4.79)$ & $120^{\circ}$ & 20.4 \\
\hline
\end{tabular}

$\left.{ }^{a}\right)$ The full-width at half-maximum (FWHM) of the peaks are given in brackets

The irradiation of the DA:HSP monolayer with UV light induces the in situ polymerization of DA at the air/liquid interface. Therefore, an emerging peak at $520 \mathrm{~nm}$ was detected, related to the formation of the red PDA polymer at the air/water interface. ${ }^{16}$ The kinetics of the interfacial polymerization has been followed in situ by monitoring the GIXD peaks along the irradiation, see Figure 2 and Table 2. A shift of the diffraction peaks is observed from $q_{\mathrm{xy}}=1.46,1.56 \AA^{-1}$ before polymerization (up to $q_{\mathrm{xy}}=1.49,1.52 \AA^{-1}$ when the polymerization is complete. These GIXD peaks are partially overlapping. This final diffraction pattern is consistent with an orthorhombic cell of $a=4.91 \AA, b=4.15 \AA$ ( $b^{\prime}=8.29 \AA$ ), $\gamma=120^{\circ}$, and an area per unit cell, $A=20.4 \AA^{2}$. The hydrophobic chains are located completely perpendicular to the interface $\left(q_{\mathrm{z}}=0\right)$. The $2 \mathrm{D}$ plot of the X-ray intensities as a function of $q_{\mathrm{xy}}$ and $q_{\mathrm{z}}$ is shown in Figure 3. The in-plane coherence length $L_{\mathrm{xy}}$ ca. $120 \AA$ can be estimated, corresponding to ca. 25 hydrophobic chains. ${ }^{32}$ Despite the shape of the domains observed by BAM retain its form and size after the polymerization, the coherence length of the organized structure decreases slightly with polymerization of the DA,. The calculated thickness of the monolayer after the polymerization, $L_{\mathrm{z}}$ is equal to ca. $28 \AA$ is in good agreement with the expected value assuming an all-trans conformation of the hydrophobic chains.

\section{Mixed Langmuir monolayer DA:SP.}

The mixed Langmuir monolayer DA:SP displays a phase transition at ca. $9.5 \mathrm{mN} / \mathrm{m}$, as no micrometric structures are observed by BAM before such transition. ${ }^{15}$ Small circular domains were observed for values of surface pressure greater than $9.5 \mathrm{mN} / \mathrm{m}$. The domains grow and shown a complex texture with further increase of the surface pressure, see Figure 4, Inset. 


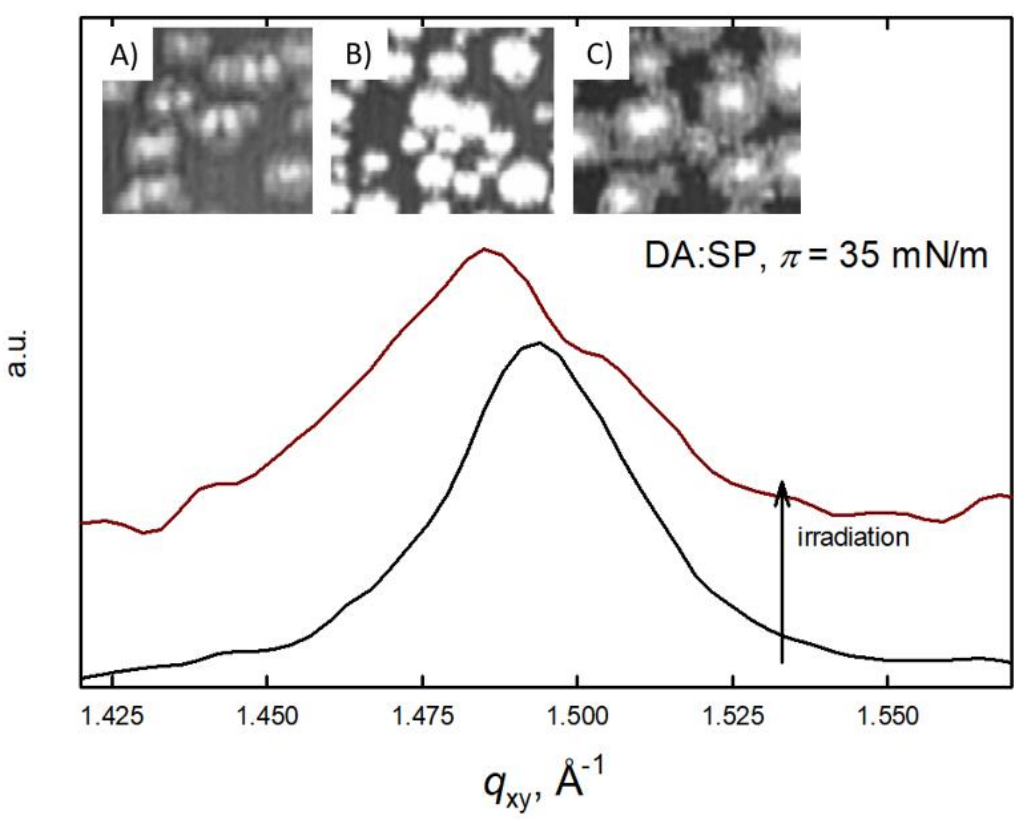

FIGURE 4. GIXD diffraction peaks for the mixed Langmuir monolayer DA:SP prior to in situ polymerization (black line) and after polymerization for the mixed Langmuir monolayer PDA:SP (red line). Both GIXD experiments were performed at $35 \mathrm{mN} / \mathrm{m}$. Inset: BAM pictures A) prior to the polymerization, B) after the polymerization, C) after expansion of the PDA:SP monolayer.

No GIXD signal was detected below $9.5 \mathrm{mN} / \mathrm{m}$, before the phase transition. However, a significant GIXD signal was detected at higher surface pressure, after the phase transition, coincidentally with the appearance of the domains. A single diffraction peak at $q_{\mathrm{xy}}=1.494 \AA^{-1}$ was obtained at $35 \mathrm{mN} / \mathrm{m}$. This diffraction pattern is consistent with a hexagonal cell of $a=b=$ $4.86 \AA,\left(b^{\prime}=8.41 \AA\right.$ ), and an area per unit cell, $A=20.4 \AA^{2}$ (see Table 3). The X-ray intensities as a function of the scattering vector components $q_{\mathrm{xy}}$ and $q_{\mathrm{z}}$ are shown in Figure 5. The hydrophobic chains are located approximately perpendicular to the interface.
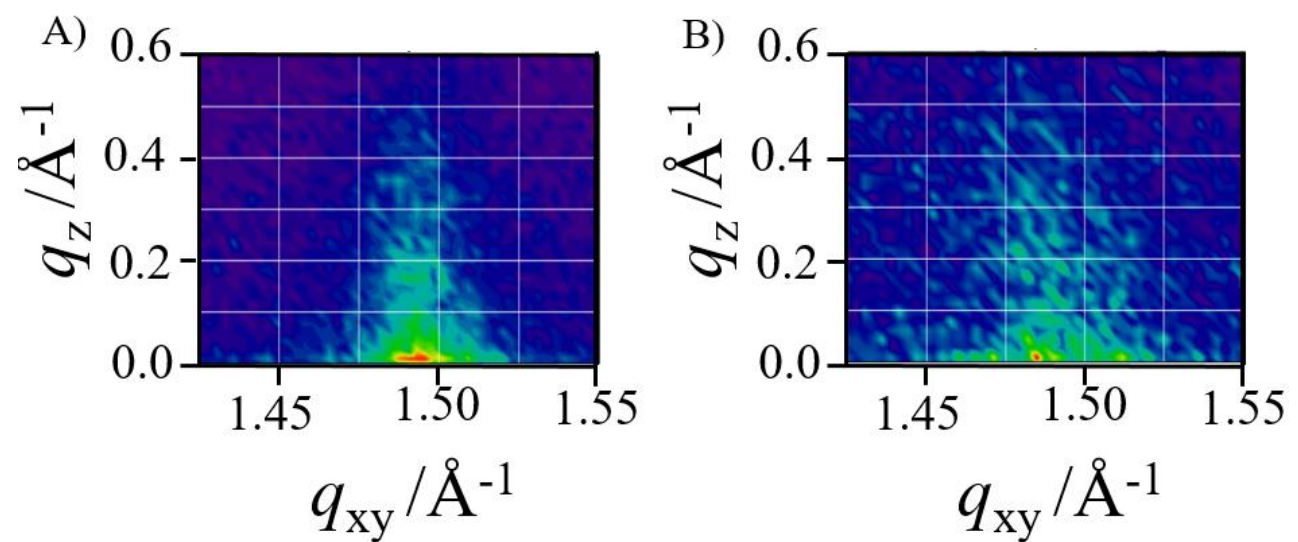
FIGURE 5. X-ray intensities versus the in-plane and out-of-plane scattering vector component $q_{\mathrm{xy}}$ and $q_{\mathrm{z}}$, for the mixed Langmuir monolayer DA:SP at $35 \mathrm{mN} / \mathrm{m} \mathrm{A}$ ) prior to in situ polymerization, B) after the in situ polymerization.

The in-plane coherence length, range at ca. 35 alkyl chains at $35 \mathrm{mN} / \mathrm{m}$, similarly to the DA:HSP monolayer, see above. The thickness of the diffracting plane $\left(L_{z}\right)$ can be estimated to ca. $22 \AA$.

The DA molecules within the mixed Langmuir monolayer DA:SP have been polymerized by irradiation with UV light. The brightness of the domains increase after the polymerization of DA, with no change in shape. The expansion of the DA:SP monolayer leads to disappearance of the domains, whereas expansion of the polymerized PDA:SP monolayer evidence stable and rigid domains, see Inset in Figure $4 .{ }^{16}$

\begin{tabular}{|c|c|c|c|c|c|}
\hline & & $a, \AA$ & $b^{\prime}(b), \AA$ & $\gamma$ & $A, \AA^{2}$ \\
\hline DA:SP & $\begin{array}{l}q_{\mathrm{xy}}=1.494(0.034) \\
q_{\mathrm{z}}=0(0.25)\end{array}$ & 4.86 & $8.41(4.86)$ & $120^{\circ}$ & 20.4 \\
\hline PDA:SP & $\begin{array}{l}q_{\mathrm{xy} 1}=1.485(0.046) \\
q_{\mathrm{xy} 2}=1.51 \\
q_{\mathrm{z} 1}=0(0.22)\end{array}$ & 4.91 & $8.30(4.79)$ & $120^{\circ}$ & 20.4 \\
\hline
\end{tabular}

A splitting of the GIXD diffraction peak after polymerization is observed, obtaining values of $q_{\mathrm{xy}}=1.485$ and $1.51 \AA^{-1}$. This diffraction pattern is consistent with an orthorhombic cell of $a=4.91 \AA, b=4.16 \AA$ ( $\left.b^{\prime}=8.32 \AA\right), \gamma=120^{\circ}$, an area per unit cell, $A=20.6 \AA^{2}$. The hydrophobic chains are located completely perpendicular to the interface.

Similarly to the DA:HSP monolayer (see above), the polymerization increased the FWHM of the Bragg peaks. Thus, the coherence length is reduced from 35 to ca. 25 after the polymerization. Moreover, the thickness of the monolayer $\left(L_{z}\right)$ can be estimated as ca. $26 \AA$, in a good agreement with an all-trans conformation. Although the diffraction spectrum evolves from a hexagonal to orthorhombic structure, a complete segregation of the individual components of the mixed monolayer is ruled out, given the observed GIXD diffraction peaks do not correspond to those peaks of the isolated components, SP and PDA.

\section{Molecular model for Langmuir monolayers containing DA prior to polymerization.}

No diffraction peaks corresponding to the isolated components have been observed for both DA:HSP and DA:SP system, which suggests the formation of mixed homogeneous Langmuir monolayers, confirming previous studies based on BAM and UV-vis reflection spectroscopy. ${ }^{15,16}$ Despite the GIXD results cannot rule out a coexistent and disordered phase, 
we note that such phase must be composed of DA and either HSP or SP in equimolar ratio, given no diffraction peaks corresponding to the isolated components have been observed.

The similar length of the hydrophobic chains of either hemicyanine dye combined with the DA molecule is beneficial for an optimum molecular packing, as commented above. Note however that the hydrophobic region of the DA molecule is composed by two segments that are tilted with respect to each other at the diacetylene group. Despite both hydrophobic segments are completely vertical respect to the air/water interface as deduced from the GIXD results, the hydrophobic segments are not coincidentally arranged on the same vertical. The hydrophobic segments are displaced with respect to each other by ca. $3.7 \AA$ from molecular modeling, see Scheme 2A. Such a conformation is hardly compatible with the HSP or SP molecules displaying a completely vertical hydrophobic chain.

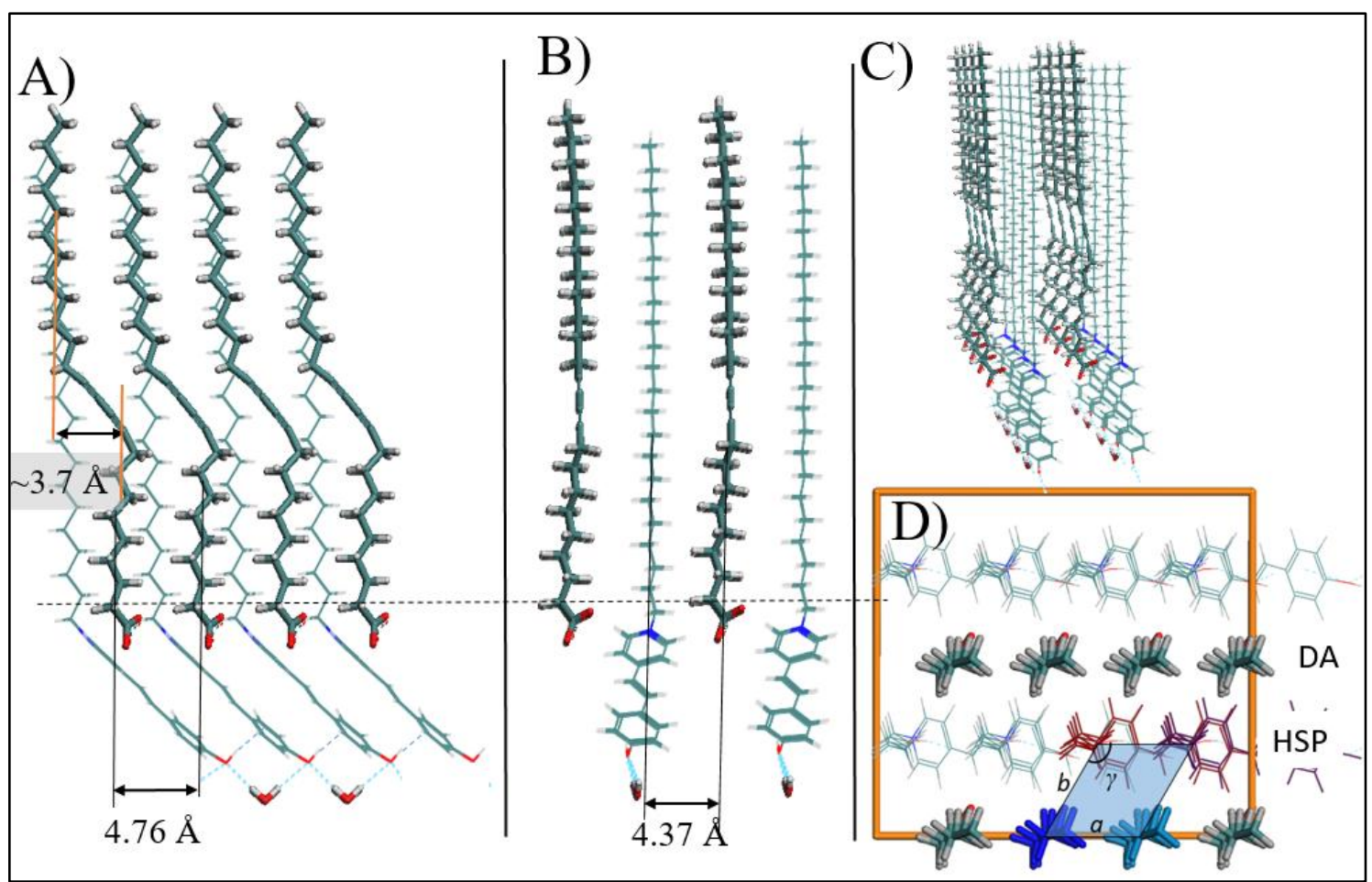

SCHEME 2. Molecular structure of the DA:HSP mixed Langmuir monolayer as obtained by Molecular Mechanics simulations. (A, B, C, D) Frontal, lateral, 3D, and top perspectives, respectively. To improve clarity in (D), certain molecules are depicted in different colors. Note that exclusively the hydrophobic chains located between the butadiene and the polar groups are drawn. The 2D periodic box used is shown in $\mathrm{D}$ ) (orange line), along with the unit cell determined from the GIXD data $\left(a=4.76 \AA, b=5.15 \AA\right.$ and $\left.\gamma=121.9^{\circ}\right)$.

The matching between the hydrophobic chains of the HSP and DA molecules has been analyzed by computer simulations based on Molecular Mechanics. A 2D periodic box containing 16 intercalated molecules ( 8 DA and 8 HSP) was built. The size of the periodic box is $4 \cdot a=19.04 \AA, 4 \cdot b \cdot \cos (\gamma-\pi / 2)=17.48 \AA$, and $\alpha=90^{\circ}$ with $\mathrm{p} 1$ symmetry, see orange line in Scheme 2D. The computational value of area per molecule is set as coincident with the experimental value. The most stable packing between DA and HSP requires alternating rows of 
the DA and HSP molecules, see Scheme 2. The 2D periodic box is also displayed in and $\gamma=121.9^{\circ}$ ), showing the good agreement between experimental and computational results.

Adjacent DA molecules are arranged with the diacetylene group and hydrophobic segments of each DA molecule in the same plane as the neighbor molecule, see scheme 2 . The molecular model accounts for the molecular aggregation of the hemicyanine group as previously described by UV-vis reflection experiments. ${ }^{16}$

Note the hemicyanine group of the HSP molecule has an $-\mathrm{OH}$ group that might contribute to the alignment of the rows of molecules through an aromatic hydrogen bond, see scheme $2 \mathrm{~A} .{ }^{33}$ Such hydrogen bond cannot be present in the DA:SP mixed monolayer given the absence of the mentioned -OH group in the hemicyanine group of SP. Yet these structural differences are not relevant as shown by the similar values of in-plane coherence length estimated from the FWHM of the Bragg peaks.

\section{Molecular model for Langmuir monolayers containing DA after the polymerization}

The size and shape of the unit cell for the mixed monolayers DA:HSP and DA:SP show significant differences, see Tables 2,3. Such differences are attributed to the subtle yet relevant modification in the polar headgroup of HSP and SP molecules. Strikingly, the size and type of unit cell of both PDA:HSP and PDA:SP monolayers after the in situ polymerization by irradiation of the monolayer with UV light are similar. Thus we conclude that the structure of the polydiacetylene impose the crystalline packing at the air/water interface even in mixed Langmuir monolayers. Note the polymerization takes place in both monolayers with an expansion of the unit cell along the $a$ axis, as well as a contraction along the $b$ axis.

The GIXD diffraction peaks for the PDA:HSP and PDA:SP monolayers point to the red form of PDA rather than the blue form in the mixed monolayers, in agreement with previous reports see Table $1 .^{18,27,16}$

3D polymerization of DA in bulk conditions exclusively occurs along of the direction of the $\boldsymbol{r}$ axis, with no polymerization along the $\boldsymbol{k}$ axis, leading to the blue polymer form, see Scheme 1D. ${ }^{19}$ The molecular arrangement of the DA molecules forming the PDA polymer on 2D mixed monolayers is significantly different to the arrangement in 3D conditions, e. g., bulk solution, although the molecular organization of the DA molecules along the $\boldsymbol{k}$ axis of the PDA polymer is identical in both $2 \mathrm{D}$ and $3 \mathrm{D}$ scenarios. However there is no possibility of polymerization along the $\boldsymbol{k}$ direction with the described mechanism. Therefore an alternative mechanism for polymerization of diacetylene in 2D has been proposed, even with alignment of the DA molecules along the $\boldsymbol{k}$ axis. ${ }^{21,20}$ This different mechanism of polymerization is based on the polymerization along the $\boldsymbol{k}$ axis in 2D, opposed to the polymerization along the $\boldsymbol{r}$ axis in $3 D .^{20,21,34}$ 
Computer simulations based on Molecular Mechanics were performed to attain

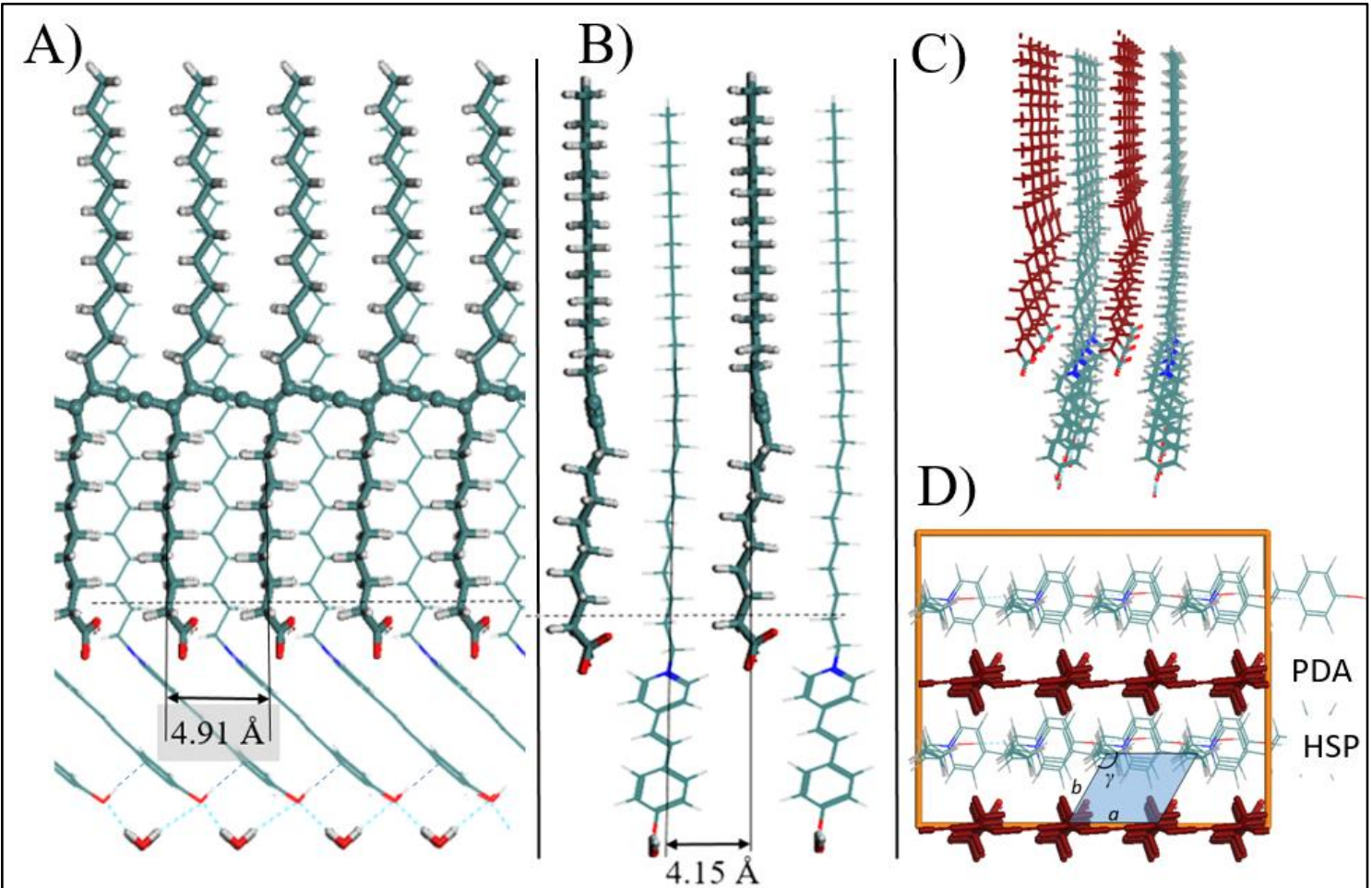

SCHEME 3. Structure of the PDA:HSP mixed monolayer obtained by Molecular Mechanics simulations. (A)-(D) are frontal, lateral, 3D, and top perspectives, respectively. For a better structure visualization, in (D), PDA is represented in red colors, and only the DA and HSP alkyl chains fraction located between the butadiene and the polar groups are drawn. Also, in (D), the 2D periodic box used is visualized (orange line), and the unit cell determined from the GIXD data is reconstructed $\left(a=4.91 \AA, b=4.79 \AA\right.$ and $\left.\gamma=120^{\circ}\right)$.

The most stable structure of PDA is shown in scheme 3. The 2D periodic box for the computer simulations is also depicted (orange line), as well as the unit cell determined from the GIXD data $\left(a=4.91 \AA, b=4.79 \AA\right.$ and $\gamma=120^{\circ}$ ). A good agreement of the unit cell described by GIXD experiments with the computational results is obtained. The structure of the PDA maintains the packing in alternating rows of PDA and either HSP or SP molecules. Similar to the scenario prior to the polymerization, the $-\mathrm{OH}$ group from the HSP polar headgroup might contribute to the correct molecular packing.

\section{Conclusions}


Polidiacetylene (PDA) is a highly relevant material in nanoscience with outstanding

\section{ACKNOWLEDGMENTS}

Support from the Ministry of Economy and Competitiveness is acknowledged through the following projects: CTQ2014-56422-P and CTQ2014-57515- C2. J.J.G.-C. acknowledges the Ministry of Economy and Competitiveness for a Ramon y Cajal contract (\#RyC-2014-14956). G.G-E. acknowledges the Ministry of Education, Culture and Sport for a predoctoral grant (Formación de Personal Universitario, FPU). We thanks Philippe Fontaine for setting the experiment on the SIRIUS beamline.

\section{REFERENCES}

(1) Park, D.-H.; Jeong, W.; Seo, M.; Park, B. J.; Kim, J.-M. Adv. Funct. Mater. 2016, 26 (4), 498.

(2) Wang, D.-E.; Wang, Y.; Tian, C.; Zhang, L.; Han, X.; Tu, Q.; Yuan, M.; Chen, S.; Wang, J. J. Mater. Chem. A 2015, 3 (43), 21690.

(3) Kim, J.; Lee, J.; Kim, W. Y.; Kim, H.; Lee, S.; Lee, H. C.; Lee, Y. S.; Seo, M.; Kim, S. Y. Nat. Commun. 2015, 6, 6959.

(4) Zhu, L.; Trinh, M. T.; Yin, L.; Zhang, Z. Chem. Sci. 2016, 7 (3), 2058. 
(5) Pattanatornchai, T.; Charoenthai, N.; Traiphol, R. J. Colloid Interface Sci. 2014, 432, 176.

(6) Yang, D.; Zou, R.; Zhu, Y.; Liu, B.; Yao, D.; Jiang, J.; Wu, J.; Tian, H. Nanoscale 2014, $6(24), 14772$.

(7) Yao, D.; Li, S.; Zhu, X.; Wu, J.; Tian, H. Chem. Commun. 2017, 2.

(8) Okaniwa, M.; Oaki, Y.; Kaneko, S.; Ishida, K.; Maki, H.; Imai, H. Chem. Mater. 2015, 27 (7), 2627.

(9) Spagnoli, S.; Briand, E.; Vickridge, I.; Fave, J.-L.; Schott, M. Langmuir 2017, 33 (6), 1419.

(10) Jonas, U.; Shah, K.; Norvez, S.; Charych, D. H. J. Am. Chem. Soc. 1999, 121 (19), 4580.

(11) Xu, Y.; Li, J.; Hu, W.; Zou, G.; Zhang, Q. J. Colloid Interface Sci. 2013, 400, 116.

(12) Giner-Casares, J. J.; Brezesinski, G.; Möhwald, H. Curr. Opin. Colloid Interface Sci. 2014, 19 (3), 176.

(13) Chen, X.; Zhou, G.; Peng, X.; Yoon, J. Chem. Soc. Rev. 2012, 41 (13), 4610.

(14) Park, H. K.; Chung, S. J.; Park, H. G.; Cho, J. H.; Kim, M.; Chung, B. H. Biosens. Bioelectron. 2008, 24 (3), 480.

(15) Ariza-Carmona, L.; Martín-Romero, M. T.; Giner-Casares, J. J.; Pérez-Morales, M.; Camacho, L. J. Phys. Chem. C 2013, 117, 21838-21848.

(16) Ariza-Carmona, L.; Martín-Romero, M. T.; Giner-Casares, J. J.; Camacho, L. J. Colloid Interface Sci. 2015, 459, 53.

(17) Enkelmann, V. Adv. Polym. Sci. 1984, 63, 91.

(18) Lifshitz, Y.; Golan, Y.; Konovalov, O.; Berman, A. Langmuir 2009, 25 (8), 4469.

(19) Wegner, G. Z. Naturforsch. B 1969, 24, 824.

(20) Okawa, Y.; Takajo, D.; Tsukamoto, S.; Hasegawa, T.; Aono, M. Soft Matter 2008, 4 (5), 1041.

(21) Takajo, D.; Inaba, A.; Sudoh, K. Langmuir 2014, 30 (10), 2738.

(22) Gaboriaud, F.; Golan, R.; Volinsky, R.; Berman, A.; Jelinek, R. Langmuir 2002, 17 (12), 3651.

(23) Fontaine, P.; Ciatto, G.; Aubert, N.; Goldmann, M. Sci. Adv. Mater. 2014, 6 (11), 2312.

(24) Hypercube: Gainesville, FL 2003,.

(25) Sun, H. J. Phys. Chem. B 1998, 102 (38), 7338.

(26) Goettgens, B. M.; Tillmann, R. W.; Radmacher, M.; Gaub, H. E. Langmuir 1992, 8 (7), 1768.

(27) Gourier, C.; Alba, M.; Braslau, A.; Daillant, J.; Goldmann, M.; Knobler, C. M.; Rieutord, F.; Zalczer, G. Langmuir 2001, No. 16, 6496.

(28) Turshatov, A. A.; Zaitsev, S. Y.; Sazonov, S. K.; Vedernikov, A. I.; Gromov, S. P.; Alfimov, M. V.; Möbius, D. Colloids Surfaces A Physicochem. Eng. Asp. 2008, 329 (12), 18 .

(29) González-Delgado, A. M.; Rubia-Payá, C.; Roldán-Carmona, C.; Giner-Casares, J. J.; Pérez-Morales, M.; Muñoz, E.; Martín-Romero, M. T.; Camacho, L.; Brezesinski, G. J. Phys. Chem. C 2010, 114, 16685.

(30) Kaganer, V. M.; Möhwald, H.; Dutta, P. Rev. Mod. Phys. 1999, 71 (3), 779.

(31) Lepère, M.; Chevallard, C.; Brezesinski, G.; Goldmann, M.; Guenoun, P. Angew. Chemie - Int. Ed. 2009, 48 (27), 5005.

(32) Daillant, J.; Alba, M. Reports Prog. Phys. 2000, 63 (10), 1725.

(33) Biedermann, F.; Schneider, H.-J. Chem. Rev. 2016, 116 (9), 5216.

(34) Ariza-Carmona, L.; Rubia-Payá, C.; García-Espejo, G.; Martín-Romero, M. T.; GinerCasares, J. J.; Camacho, L. Langmuir 2015, 31 (19), 5333.

\section{GRAPHICAL ABSTRACT}

